\title{
Relationship of High-Molecular-Weight Adiponectin Levels to Visceral Fat Accumulation in Hemodialysis Patients
}

\author{
Noriko Tamei ${ }^{1,2}$, Tetsuya Ogawa ${ }^{1}$, Hideki Ishida $^{2}$, Yoshitaka Ando ${ }^{2}$ and Kosaku Nitta ${ }^{1}$
}

\begin{abstract}
Objective High molecular weight adiponectin (HMW ADPN) plays an important role in the regulation of insulin resistance and atherogenic processes. However, the role of HMW ADPN remains to be determined in hemodialysis (HD) patients.

Patients and Methods We measured serum HMW ADPN in 49 HD patients (age: $62.4 \pm 12.0$ years, time on HD: $8.4 \pm 6.4$ years, male/female=30/19), and examined the association between HMW ADPN and visceral fat area (VFA) estimated by abdominal CT scans.

Results Serum HMW ADPN concentrations were weakly and inversely correlated with serum TG ( $r=-$ 0.271, $\mathrm{p}=0.0598)$, but significantly and positively correlated with HDL cholesterol $(\mathrm{r}=0.392, \mathrm{p}=0.0050)$. Serum HMW ADPN levels were positively correlated with BMI ( $\mathrm{r}=0.472, \mathrm{p}=0.0084)$ in male patients, and the HMW ADPN levels were positively correlated with serum levels of HDL cholesterol $(\mathrm{r}=0.514, \mathrm{p}=0.0243$ ) and TG $(r=0.605, p=0.0061)$. The regression coefficient between VFA and HMW ADPN was -0.491 ( $\mathrm{p}<$ 0.003). Multiple stepwise regression analyses showed that VFA was the most significant and independent determinant of serum HMW ADPN concentration.

Conclusion These findings suggest that HMW ADPN may be inversely associated with visceral fat accumulation in HD patients.
\end{abstract}

Key words: adiponectin, hemodialysis, visceral fat area, body mass index, HDL cholesterol

(Inter Med 49: 299-305, 2010)

(DOI: 10.2169/internalmedicine.49.2905)

\section{Introduction}

Adipose tissue is now considered to be a secretory organ for physiologically active substances that have been generically designated as 'adipocytokines'. Adiponectin (ADPN) is a collagen-like plasma protein of adipose tissue that is known to play an important role in the development of metabolic syndrome $(1,2)$. ADPN has both anti-atherogenic and anti-inflammatory properties. Adiponectin protects against the development of systolic dysfunction after myocardial infarction in adiponectin-deficient mice $(3,4)$. In addition, ADPN is a key regulator of albuminuria to modulate oxidant stress in podocytes in adiponectin-deficient mice (5). These findings have provided data to support the notion that ADPN is essentially cardio- and renal-protective agent.

Odamaki et al (6) previously reported that HD patients tend to have excessive accumulation of intra-abdominal adipose tissue despite their lower body mass index (BMI) relative to healthy subjects. They also demonstrated that visceral fat accumulation correlated well with serum lipid abnormalities and atherosclerotic index in these patients. Although cardiovascular diseases resulting from atherosclerotic complications are a major cause of death in HD patients, it is unclear how the accumulation of intra-abdominal fat influences the progression of atherosclerosis in HD patients.

ADPN circulates in three major forms: as trimers (low molecular weight), hexamers (medium molecular weight), and as 12-mers or 18-mers (high molecular weight [HMW]). These major forms of ADPN differently activate the signaling pathways in the target tissues (1). HMW ADPN is the only multimetric form that prevents apoptosis of cultured human endothelial cells (7). Decreased HMW ADPN is closely associated with the development of type 2 diabe-

${ }^{1}$ Department of Medicine, Kidney Center, Tokyo Women's Medical University, Tokyo and ${ }^{2}$ Hidaka Hospital, Gunma Received for publication September 15, 2009; Accepted for publication October 19, 2009 Correspondence to Dr. Tetsuya Ogawa, togawa@kc.twmu.ac.jp 
tes (8). Hara et al (9) also demonstrated that the ratio of HMW to total ADPN in plasma is more useful in the prediction of insulin resistance and metabolic syndrome when compared with total ADPN. The HMW-to-total ADPN ratio is also inversely correlated with insulin resistance in men with normal glucose tolerance.

Circulating ADPN concentration is higher in hemodialysis (HD) patients than in healthy subjects, since plasma ADPN level is dependent on glomerular filtration rate (10). Moreover, a low plasma ADPN level appears to be a strong predictor of cardiovascular events in HD patients (11). A 1.2fold increase in plasma HMW ADPN is found in hemodialysis (HD) patients when compared with healthy subjects (12). However, the relation of increased HMW ADPN to visceral fat accumulation in HD patients remains to be determined.

The aim of the present study was to measure plasma HMW ADPN levels and to examine the association of the serum HMW ADPN levels with visceral fat accumulation and clinical parameters in chronic HD patients.

\section{Patients and Methods}

\section{Patients}

All subjects were treated at the Dialysis Unit of Hidaka Hospital for more than 6 months, and received standardized HD prescriptions $(500 \mathrm{~mL} / \mathrm{min}$ dialysate flow; $250-300 \mathrm{~mL} /$ min blood flow; 4 hours dialysis per session; 3 sessions per week). Of all HD patients, 49 patients (age: 62.4 \pm 12.0 years, time on HD: $8.4 \pm 6.4$ years, male/female=30/19) gave their informed consent and completed the study. The dialysis potassium concentration was $2.0 \mathrm{mEq} / \mathrm{L}$ and the $\mathrm{Ca}$ concentration was $3.0 \mathrm{mEq} / \mathrm{L}$. Blood pressure was recorded three times with a brachial sphygmomanometer, after the subject had rested in the supine position for at least $10 \mathrm{~min}$ and the average value of the three measurements was adopted. Hypertension was defined as systolic blood pressure (SBP) $\geq$ $140 \mathrm{mmHg}$ and/or diastolic blood pressure (DBP) $\geq 90$ $\mathrm{mmHg}$, or use of antihypertensive medication. Diabetes mellitus (DM) was defined as either fasting glucose $>126 \mathrm{mg} /$ $\mathrm{dL}$, random glucose $>200 \mathrm{mg} / \mathrm{dL}$, or use of insulin or antidiabetic medication. All measurements as listed below were performed after dialysis session except blood sampling. Information of antihypertensive medications were obtained from medical charts and analyzed. None of the patients was treated with thiazolidinedione. This study was in compliance with the Declaration of Helsinki and was in agreement with the guidelines approved by the ethics committee of our institution and informed consent was obtained from each patient.

\section{CT scans}

The multi-detector-row CT examination was performed using GE LightSpeed. An index image was obtained before scanning, and the umbilicus to L4-5 level was identi- fied (13). The thickness of a slice was $10 \mathrm{~mm}$. The radiographic images were digitally scanned for analysis by a personal computer. The abdominal visceral fat mass area (VFA) at this level correlates extremely well with whole intraabdominal fat content ( $\mathrm{r}=0.94)$. Horizontal image was obtained at $400 \mathrm{~mA}$ and $120 \mathrm{kVp}$ with a scan time of $1.0 \mathrm{~s}$. The range of CT values covers optimal CT numbers for fat tissue, from -150 to -40 . Data were stored and analyzed using a GE advantage workstation Ver 4.0.

\section{Laboratory studies}

Serum urea nitrogen, creatinine, calcium, phosphorous, albumin, total cholesterol, high-density lipoprotein (HDL)cholesterol, low-density lipoprotein (LDL)-cholesterol triglyceride, C-reactive protein (CRP), and hemoglobin were measured by standard laboratory techniques using an autoanalyzer. Body mass index (BMI) was calculated dividing dry weight $(\mathrm{kg})$ by body height $(\mathrm{m})^{2}$. Intact parathyroid hormone (iPTH) was measured by immunoradiometric assay. Total and HMW ADPN were measured by using commercially available human HMW ADPN ELISA kits (Fujirebio, Tokyo, Japan) and total ADPN (Otsuka Pharmaceuticals, Tokyo, Japan) as previously described (14-16). The mean HMW/total ADPN ratio was calculated. The reference group included 42 healthy control subjects matched to HD patients for gender (males/females, 24/18) and age (59.3 \pm 12.7 years).

\section{Statistical analysis}

Values were expressed as the means \pm SD. Differences between two groups were analyzed by an unpaired Student $\mathrm{t}$ test following the analysis of variance. $p$ values $<0.05$ were considered statistically significant. Pearson's correlation coefficient was used to assess the association between continuous variables. Multivariate linear regression was performed to determine the factors related to the serum HMW ADPN levels using variables such as age, HD vintage, BMI, HDLcholesterol, triglyceride and log-transformed CRP. Stepwise backward elimination was used, beginning with the variable with the highest P-value. We also examined determinants of serum HMW ADPN with multiple stepwise regression analysis. We analyzed four parameters: BMI, serum levels of HDL-cholesterol and triglycerides, and VFA. All statistical calculations were performed with StatView 5J software (SAS Institute, Cary, NC, USA).

\section{Results}

\section{Baseline patient profiles}

The baseline profiles of the patients are shown in Table 1. At the assessment, the age of the patients was $62.4 \pm 12.0$ (32-86) years, with a mean HD duration of $8.4 \pm 6.4$ (0.624.8 ) years $($ male/female $=30 / 19)$. The underlying kidney diseases were chronic glomerulonephritis $(n=29)$, and diabetic nephropathy $(\mathrm{n}=20)$. The VFA was $85.2 \pm 57.8 \mathrm{~cm}^{2}$. The 
Table 1. Baseline Characteristics of the Study Population $(n=49)$

\begin{tabular}{lc}
\hline Clinical variables & \\
\hline Hypertension $(\%)$ & $20(81.6)$ \\
Diabetes $(\%)$ & $3.7 \pm 0.3$ \\
Albumin $(\mathrm{g} / \mathrm{dL})$ & $10.2 \pm 0.9$ \\
Hemoglobin $(\mathrm{g} / \mathrm{dL})$ & $0.26 \pm 0.41$ \\
C-reactive protein $(\mathrm{mg} / \mathrm{dL})$ & $145.7 \pm 25.9$ \\
Total cholesterol $(\mathrm{mg} / \mathrm{dL})$ & $45.3 \pm 13.0$ \\
HDL $(\mathrm{mg} / \mathrm{dL})$ & $76.9 \pm 22.5$ \\
LDL $(\mathrm{mg} / \mathrm{dL})$ & $105.4 \pm 89.6$ \\
Triglyceride $(\mathrm{mg} / \mathrm{dL})$ & $8.8 \pm 0.8$ \\
Calcium $(\mathrm{mg} / \mathrm{dL})$ & $5.5 \pm 1.2$ \\
Phosphate $(\mathrm{mg} / \mathrm{dL})$ & $258.7 \pm 131.7$ \\
intact PTH $(\mathrm{pg} / \mathrm{mL})$ & $26.9 \pm 20.3$ \\
HMW ADPN $(\mu \mathrm{g} / \mathrm{mL})$ & $40.3 \pm 20.9$ \\
Total ADPN $(\mu \mathrm{g} / \mathrm{mL})$ & $0.57 \pm 0.18$ \\
H/T ADPN ratio & $85.2 \pm 57.8$ \\
Visceral fat area $\left(\mathrm{cm}{ }^{2}\right)$ & \\
\hline
\end{tabular}

HDL, high-density lipoprotein cholesterol; LDL, low-density lipoprotein cholesterol; PTH, parathyroid hormone; HMW ADPN, high molecular weight adiponectin; H/T ADPN ratio, high molecular/total adiponectin ratio.

mean serum levels of total ADPN and HMW ADPN were $40.3 \pm 20.9 \mu \mathrm{g} / \mathrm{mL}$ and $26.9 \pm 20.3 \mu \mathrm{g} / \mathrm{mL}$, respectively. The mean HMW/total ADPN ratio was $0.57 \pm 0.18$. The total ADPN and HMW ADPN concentrations were significantly higher in HD patients than in control subjects $(12.3 \pm 7.2 \mu \mathrm{g} /$ $\mathrm{mL}, 7.5 \pm 5.1 \mu \mathrm{g} / \mathrm{mL})$. Angiotensin-converting enzyme inhibitors and angiotensin receptor blockers were prescribed in 32 patients. The serum HMW ADPN levels were not significantly different in patients with and without these agents $(27.6 \pm 21.8 \mu \mathrm{g} / \mathrm{mL}$ vs. $22.6 \pm 16.8 \mu \mathrm{g} / \mathrm{mL})$.

Table 2 shows a comparison of clinical and biochemical profiles between male and female HD patients. The mean serum levels of total ADPN, HMW ADPN and the HMW/ total ADPN ratio tended to be higher in female patients, but these did not reach significance. Serum HMW ADPN levels were positively correlated with BMI $(r=0.472, p=0.0084)$ in male patients, and the HMW ADPN levels were positively correlated with serum levels of HDL-cholesterol ( $\mathrm{r}=0.514$, $\mathrm{p}=0.0243)$ and TG $(\mathrm{r}=0.605, \mathrm{p}=0.0061)$.

Table 3 shows the comparison of clinical and biochemical profiles between diabetic and non-diabetic HD patients. Diabetic patients were younger than non-diabetic patients. There was no significant difference in gender, dialysis vintage, BMI, diastolic blood pressure, hemoglobin, serum levels of albumin, CRP, total cholesterol, HDL cholesterol, LDL cholesterol, triglyceride, $\mathrm{Ca}$ and $\mathrm{P}$ in both groups. The systolic blood pressure, serum levels of total ADPN, serum HMW ADPN and HMW/total ADPN ratio were higher in diabetic patients. The serum intact PTH level was lower in diabetic patients.

As shown in Table 4, HMW ADPN was not correlated with age $(\mathrm{r}=-0.123, \mathrm{p}=0.4009)$, HD vintage $(\mathrm{r}=-0.068, \mathrm{p}=$ $0.6439)$, and BMI ( $r=-0.183, p=0.2096)$. There was no difference in HMW ADPN adjusted for BMI between diabetic and non-diabetic patients. Serum HMW ADPN concentrations were weakly and inversely correlated with serum TG ( $r=-0.271, p=0.0598)$, but significantly and positively correlated with HDL-cholesterol ( $r=0.392, \mathrm{p}=0.0050$; Fig. 1).

Since HMW ADPN is released from fat cells, simple and multiple regression analyses were performed to examine which part of fat was the major determinant of serum ADPN as shown in Table 4. The regression coefficient between VFA and HMW ADPN was -0.491 ( $\mathrm{p}=0.0034$; Fig. 2). Multiple stepwise regression analysis showed that VFA was the most significant and independent determinant of serum HMW ADPN concentration (Table 5). Serum logtransformed CRP was not correlated with HMW ADPN ( $r=-$ $0.0060, \mathrm{p}=0.6814$ ) in the present study.

\section{Discussion}

Serum or plasma ADPN is demonstrated to be associated with clinical parameters of cardiovascular damage in the general population. Several studies have recently shown that serum ADPN level is inversely correlated with BMI or fat mass, and that ADPN levels are low in obese persons (17) and type 2 diabetic mellitus patients (18). In addition, plasma ADPN concentration rises with loss of body weight in humans (19). Truncal fat can be divided into subcutaneous fat and visceral fat, and accumulation of the latter appears to strongly suppress the secretion of ADPN from adipocytes. Moreover, the secretion of ADPN might be decreased when visceral fat cells become hypertrophic (20). 
Table 2. Comparison of Clinical and Biochemical Profiles between Male and Female Hemodialysis Patients

\begin{tabular}{lccc}
\hline Clinical variables & Male patients & Female patients & p-value \\
\multicolumn{1}{c}{$\mathrm{n}$} & 30 & 19 & \\
\hline BMI $\left(\mathrm{kg} / \mathrm{m}^{2}\right)$ & $21.1 \pm 3.0$ & $22.4 \pm 6.3$ & 0.0611 \\
Total cholesterol $(\mathrm{mg} / \mathrm{dL})$ & $140.4 \pm 23.8$ & $154.1 \pm 27.4$ & 0.0715 \\
HDL $(\mathrm{mg} / \mathrm{dL})$ & $43.0 \pm 11.3$ & $48.8 \pm 15.0$ & 0.1287 \\
LDL $(\mathrm{mg} / \mathrm{dL})$ & $75.1 \pm 21.8$ & $79.8 \pm 21.7$ & 0.4752 \\
Triglyceride $(\mathrm{mg} / \mathrm{dL})$ & $102.2 \pm 108.3$ & $110.5 \pm 49.5$ & 0.7269 \\
HMW ADPN $(\mu \mathrm{g} / \mathrm{mL})$ & $22.6 \pm 17.1$ & $33.7 \pm 23.4$ & 0.0611 \\
Total ADPN $(\mu \mathrm{g} / \mathrm{mL})$ & $35.3 \pm 16.7$ & $48.2 \pm 24.7$ & 0.0552 \\
H/T ADPN ratio & $0.53 \pm 0.17$ & $0.63 \pm 0.20$ & 0.0793 \\
Visceral fat area $\left(\mathrm{cm}^{2}\right)$ & $93.3 \pm 56.2$ & $73.4 \pm 59.4$ & 0.2409 \\
\hline
\end{tabular}

HDL, high-density lipoprotein cholesterol; LDL, low-density lipoprotein cholesterol; PTH, parathyroid hormone; HMW ADPN, high molecular weight adiponectin; H/T ADPN ratio, high molecular/total adiponectin ratio.

Table 3. Comparison of Clinical and Biochemical Profiles between Diabetic and Non-diabetic Hemodialysis Patients

\begin{tabular}{lccc}
\hline Clinical variables & Non-diabetic patients & Diabetic patients & \\
\multicolumn{1}{c}{$\mathrm{n}$} & 29 & 20 & p-value \\
\hline Age (years) & $65.1 \pm 13.4$ & $58.6 \pm 8.5$ & 0.0422 \\
Male gender $(\mathrm{n})$ & $17(58.6 \%)$ & $13(65.0 \%)$ & 0.6605 \\
Vintage $($ years $)$ & $9.81 \pm 6.52$ & $6.24 \pm 5.70$ & 0.0534 \\
BMI $\left(\mathrm{kg} / \mathrm{m}^{2}\right)$ & $21.9 \pm 5.2$ & $21.1 \pm 3.4$ & 0.5115 \\
Systolic blood pressure $(\mathrm{mmHg})$ & $148.4 \pm 14.5$ & $158.6 \pm 10.6$ & 0.0099 \\
Diastolic blood pressure $(\mathrm{mmHg})$ & $73.6 \pm 13.8$ & $79.0 \pm 5.4$ & 0.0668 \\
Albumin $(\mathrm{g} / \mathrm{dL})$ & $3.74 \pm 0.24$ & $3.69 \pm 0.33$ & 0.4925 \\
Hb $(\mathrm{g} / \mathrm{dL})$ & $10.3 \pm 1.0$ & $10.1 \pm 0.7$ & 0.5455 \\
CRP $(\mathrm{mg} / \mathrm{dL})$ & $0.24 \pm 0.39$ & $0.28 \pm 0.44$ & 0.7089 \\
Total cholesterol $(\mathrm{mg} / \mathrm{dL})$ & $145.9 \pm 25.6$ & $145.5 \pm 27.0$ & 0.9550 \\
HDL $(\mathrm{mg} / \mathrm{dL})$ & $42.9 \pm 10.4$ & $48.7 \pm 15.7$ & 0.1576 \\
LDL $(\mathrm{mg} / \mathrm{dL})$ & $80.4 \pm 22.8$ & $71.9 \pm 21.7$ & 0.1971 \\
Triglyceride $(\mathrm{mg} / \mathrm{dL})$ & $104.7 \pm 44.6$ & $106.5 \pm 131.7$ & 0.9524 \\
Ca $(\mathrm{mg} / \mathrm{dL})$ & $8.85 \pm 0.89$ & $8.63 \pm 0.54$ & 0.2788 \\
P (mg/dL) & $5.62 \pm 0.89$ & $5.30 \pm 1.50$ & 0.4086 \\
intact PTH $(\mathrm{pg} / \mathrm{mL})$ & $302.4 \pm 135.0$ & $195.3 \pm 99.0$ & 0.0040 \\
HMW ADPN $(\mu \mathrm{g} / \mathrm{mL})$ & $19.6 \pm 16.2$ & $37.5 \pm 21.4$ & 0.0016 \\
Total ADPN $(\mu \mathrm{g} / \mathrm{mL})$ & $35.0 \pm 19.0$ & $48.0 \pm 21.6$ & 0.0313 \\
H/T ADPN ratio & $0.51 \pm 0.15$ & $0.67 \pm 0.19$ & 0.0018 \\
Visceral fat area $\left(\mathrm{cm}{ }^{2}\right)$ & $93.3 \pm 56.2$ & $73.4 \pm 59.4$ & 0.2409 \\
\hline
\end{tabular}

HDL, high-density lipoprotein cholesterol; LDL, low-density lipoprotein cholesterol; PTH, parathyroid hormone; HMW ADPN, high molecular weight adiponectin; H/T ADPN ratio, high molecular/total adiponectin ratio.

In the present study, we measured serum HMW ADPN concentration in HD patients and investigated the correlation with various clinical parameters. Although serum HMW ADPN levels in HD patients were significantly higher than those in healthy subjects, HMW ADPN level was inversely associated with VFA in HD patients. Multiple regression analysis showed that VFA was the most predictive factor on serum HMW ADPN levels. Serum HMW ADPN levels also showed a significant positive correlation with HDLcholesterol value. These results were almost identical to those in previous reports in healthy subjects (21) and type 2 diabetic males (22).

The present study showed that serum HMW ADPN levels were higher in diabetic patients than in non-diabetic patients 
Table 4. Correlation between Serum HMW ADPN Levels and Clinical Parameters in Patients on Chronic Hemodialysis

\begin{tabular}{lcc}
\hline & $\mathrm{r}$ & $\mathrm{p}$ value \\
\hline Age & -0.123 & 0.4009 \\
Vintage & -0.068 & 0.6439 \\
BMI & -0.183 & 0.2096 \\
Total cholesterol & -0.021 & 0.8879 \\
Triglyceride & -0.271 & 0.0598 \\
HDL-C & 0.392 & 0.0050 \\
Log-transformed CRP & -0.0600 & 0.6814 \\
Visceral fat area $\left(\mathrm{cm}^{2}\right)$ & -0.491 & 0.0003
\end{tabular}

BMI, body mass index; HDL-C, high-density lipoprotein-cholesterol; CRP, C-reactive protein

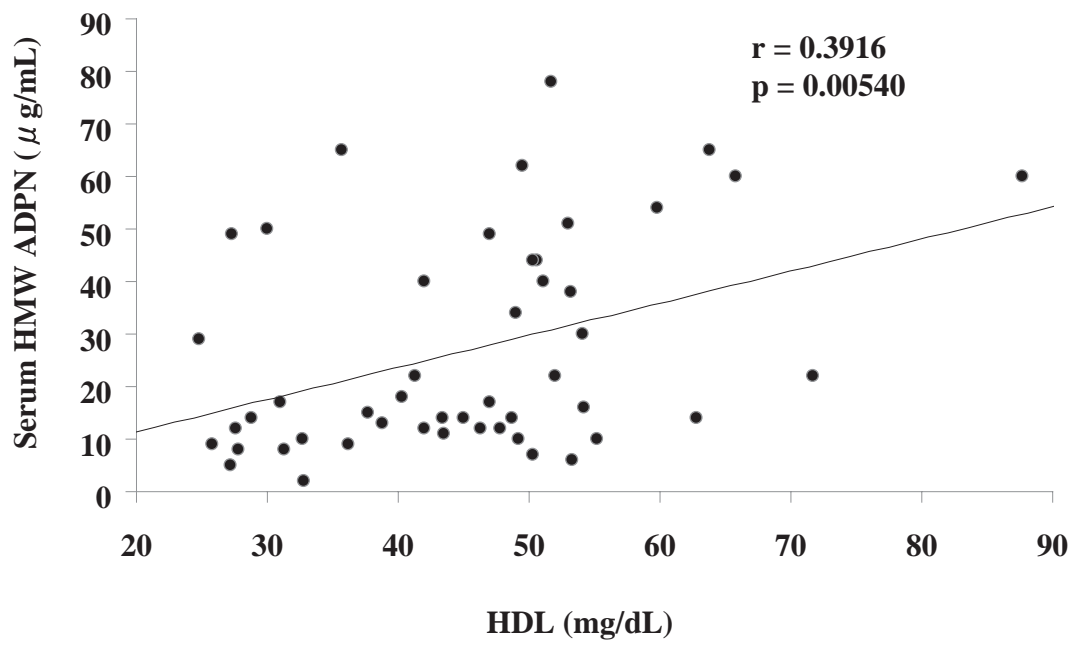

Figure 1. Correlation between high molecular weight (HMW) adiponectin (ADPN) and high-density lipoprotein (HDL) cholesterol in hemodialysis patients.

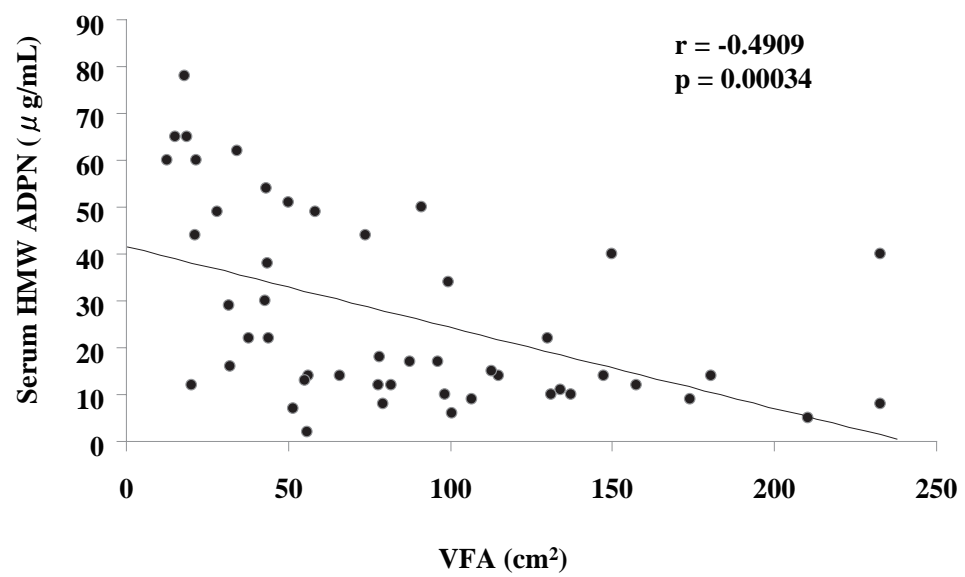

Figure 2. Correlation between high molecular weight (HMW) adiponectin (ADPN) and visceral fat area (VFA) in hemodialysis patients.

undergoing HD therapy. Previous studies have suggested that HMW ADPN may be the active form of this protein because changes in the ratio of HMW to total ADPN after treatment with a thiazolidione, but not those of total ADPN, were associated with improvement of hepatic insulin sensitivity (23) or glycemic control (24). Although the serum
ADPN level is generally believed to remain stable in vivo, it seems that HMW ADPN is affected by glucose intake in persons with impaired glucose tolerance (25). It is noteworthy that serum ADPN levels are increased in type 1 diabetes patients (26). Serum glucose or insulin concentration is likely to be a possible regulator of circulating ADPN levels. 
Table 5. Multiple Stepwise Regression Analysis for Determinant of Serum HMW ADPN Concentrations

\begin{tabular}{lll}
\hline Independent variable & F-value & p-value \\
\hline Serum HDL-cholesterol $(\mathrm{mg} / \mathrm{dL})$ & 8.512 & 0.0054 \\
Visceral fat area $\left(\mathrm{cm}^{2}\right)$ & 14.922 & 0.0003 \\
\hline
\end{tabular}

Among them, hyperglycemia is a common feature of type 1 and 2 diabetes. Therefore, hyperglycemia itself is not a critical determinant for circulating ADPN levels in vivo.

Visceral fat accumulation is considered to be upstream of the metabolic syndrome of obesity, impaired glucose tolerance, hypertension, dyslipidemia and atherosclerosis. A strong association has been demonstrated between visceral fat accumulation and atherosclerosis in the general population $(27,28)$ and HD patients (29). Recently, Yamauchi et al (30) reported that visceral fat is associated with the prevalence of carotid atherosclerosis in HD patients. Among various mechanisms by which visceral fat accumulation induces atherosclerosis, hypoadiponectinemia may play an important role. Hypoadiponectinemia has been reported to be a risk factor for cardiovascular disease. Zoccali et al (11) demonstrated that the group with lower plasma ADPN concentration had a higher rate of subsequent cardiovascular events in HD patients during a 31-month observation period.

Reduced ADPN is correlated with carotid arterial stiffness in type 2 diabetic patients (31). An inverse relationship between plasma ADPN and aortic pulse wave velocity is also found in hypertensive subjects (32). There is a greater decrease in HMW and the HMW-to-total ratio in diabetic men with coronary artery disease (CAD) than those without (33). In a report of a 7-year follow-up in CAD patients, the HMW ADPN level was inversely associated with the severity of CAD, and was a strong predictor of secondary cardiovascular events (34). In contrast, it remains unclear whether reduced ADPN is similarly related to CAD in HD patients.
In HD patients, the percentile of the HMW formed in total ADPN has been demonstrated to be much smaller in patients with ischemic heart disease than those without (35). In contrast, a cross-sectional observation failed to demonstrate a causative relationship between ADPN levels and the prevalence of vascular disease in dialysis patients (36). ADPN was also reported not to be associated with carotid atherosclerosis in HD patients (37). These findings suggest that reduction in serum HMW ADPN may be associated with advanced atherosclerosis in HD patients.

There are several limitations in this study. First, we assessed the association of HMW ADPN with clinical parameters in a cross-sectional fashion, so our observation may mask possible cause-effect relations. Second, this study was a single-center study analyzing a small number of patients. Third, we provided few potential mechanisms how HMW ADPN and HDL-cholesterol are positively related in this study. Finally, this was an observational study and, as such, patients differed with respect to a few characteristics.

In summary, we found that serum HMW ADPN was inversely associated with visceral fat accumulation in HD patients. In addition, serum HMW ADPN showed close relationships with HDL-cholesterol. These findings suggest that the regulation of HMW ADPN production by visceral fat still exists in HD patients, and this visceral fat-HMW ADPN axis appears to affect the lipid profile. The results of this study are consistent with an association of visceral fat accumulation and progression of atherosclerotic vascular disease in HD patients.

\section{References}

1. Kadowaki T, Yamauchi T, Kubota N, Hara K, Ueki K, Tobe K. Adiponectin and adiponectin receptors in insulin resistance, diabetes, and the metabolic syndrome. J Clin Invest 116: 1784-1792, 2006.

2. Matsuzawa Y, Funahashi T, Kihara S, Shimomura I. Adiponectin and metabolic syndrome. Arterioscler Thromb Vasc Biol 24: 2933, 2004.

3. Funahashi T, Matsuzawa Y. Metabolic syndrome: Clinical concept and molecular basis. Ann Med 39: 482-494, 2007.

4. Shibata R, Izumiya Y, Sato K, et al. Adiponectin protects against the development of systolic dysfunction following myocardial infarction. J Mol Cell Cardiol 42: 1065-1074, 2007.

5. Sharma K, Ramachandrarao S, Qiu G, et al. Adiponectin regulates albuminuria and podocyte function. J Clin Invest 118: 1645-1656, 2008.

6. Odamaki M, Furuya R, Ohkawa S, et al. Altered abdominal fat distribution and its association with the serum lipid profile in nondiabetic heamodialysis patients. Nephrol Dial Transplant 14: 24272432, 1999.

7. Kobayashi H, Ouchi N, Kihara S, et al. Selective suppression of endothelial cell apoptosis by the high molecular weight form of adiponectin. Circ Res 94: e27-e31, 2004.

8. Nakashima R, Kamei N, Yamane K, Nakanishi S, Nakashima A, Kohno N. Decreased total and high molecular weight adiponectin are independent risk factors for the development of type 2 diabetes in Japanese-Americans. J Clin Endocrinol Metab 91: 38733877, 2006.

9. Hara K, Horikoshi M, Yamauchi T, et al. Measurement of the high molecular weight form of adiponectin in plasma is useful for the prediction of insulin resistance and metabolic syndrome. Diabetes Care 29: 1357-1362, 2006.

10. Stenvinkel P, Marchlewska A, Pecoits-Filho R, et al. Adiponectin in renal disease: Relationship to phenotype and genetic variation in the gene encoding adiponectin. Kidney Int 65: 274-281, 2004.

11. Zoccali C, Mallamaci F, Tripepi G, et al. Adiponectin, metabolic risk factors, and cardiovascular event among patients with endstage renal disease. J Am Soc Nephrol 13: 134-141, 2002.

12. Shen YY, Charlesworth JA, Kelly JJ, Loi KW, Peake PW. Upregulation of adiponectin, its isoforms and receptors in end-stage kidney disease. Nephrol Dial Transplant 22: 171-178, 2007. 
13. Sawara $Y$, Takei $T$, Uchida K, Tsuchiya K, Nitta K. Metabolic syndrome and anthropometric factors in Japanese patients with chronic kidney disease. Heart Vessles 24: 199-203, 2009.

14. Takahashi M, Otsubo S, Uchida K, Yumura W, Nitta K. Association between serum adiponectin levels and arteriosclerosis in IgA nephropathy patients. Intern Med 46: 453-459, 2007.

15. Okano K, Ohba T, Matsugami $K$, Uchida $K$, Nitta $K$, Kabaya $T$. Analysis of plasma adipocytokines related to clinical and laboratory data in the maintenance hemodialysis patients. Intern Med $\mathbf{4 7}$ 1379-1386, 2008.

16. Iwasa $Y$, Otsubo $S$, Ishizuka $T$, Uchida $K$, Nitta $K$. Influence of serum high-molecular weight and total adiponectin on arteriosclerosis in IgA nephropathy patients. Nephron Clin Pract 108: c226c232, 2008.

17. Arita $Y$, Kihara S, Ouchi N, et al. Paradoxical decrease of an adipose-specific protein, adiponectin, in obesity. Biochem Biophys Res Commun 257: 79-93, 1999.

18. Yatagai $T$, Nagasaka $S$, Taniguchi $A$, et al. Hypoadiponectinemia is associated with visceral fat accumulation and insulin resistance in Japanese men with type 2 diabetes mellitus. Metabolism 52: 1274-1278, 2003.

19. Yang WS, Lee WJ, Funahashi T, et al. Weight reduction increases plasma levels of an adipose-derived anti-inflammatory protein, adiponectin. J Clin Endocrinol Metab 86: 3815-3819, 2001.

20. Yamauchi T, Kamon J, Waki H, et al. The mechanisms by which both heterozygous peroxisome proliferator-activated receptor gamma (PPARgamma) deficiency and PPARgamma agonist improve insulin resistance. J Biol Chem 276: 41245-41254, 2001.

21. Cnop M, Havel PJ, Utzschneider KM, et al. Relationship of adiponectin to body fat distribution, insulin sensitivity and plasma lipoproteins: evidence for independent roles of age and sex. Diabetologia 46: 459-469, 2003.

22. Hotta K, Funahashi T, Arita Y, et al. Plasma concentrations of a novel, adipose-specific protein, adiponectin, in type 2 diabetic patients. Arterioscler Thromb Vasc Biol 20: 1595-1599, 2000.

23. Tarui S, Tokunaga K, Fujioka S, Matsuzawa Y. Visceral fat obesity: anthropological and pathophysiological aspects. Int J Obes 15 (suppl 2): 1-8, 1991.

24. Pajvani UB, Hawkins M, Combs TP, et al. Complex distribution, not absolute amount of adiponectin, correlates with thiazolidinedione-mediated improvement in insulin sensitivity. J Biol Chem 279: 12152-12162, 2004.

25. Aso Y, Yamamoto R, Suetsugu M, et al. Comparison of the effects of pioglitazone and voglibose on circulating total and high- molecular-weight adiponectin, and on two fibrinolysis inhibitors, in patients with type 2 diabetes. Diabet Med 24: 962-968, 2007.

26. Imagawa A, Funahashi T, Nakamura $T$, et al. Eleveted serum concentration of adipose-derived factor, adiponectin, in patients with type 1 diabetes. Diabetes Care 25: 1665-1666, 2002.

27. Basu R, Pajvani UB, Rizza RA, Scherer PE. Selective downregulation of the high molecular weight form of adiponectin in hyperinsulinemia and in type 2 diabetes: differential regulation from nondiabetic subjects. Diabetes 56: 2174-2177, 2007.

28. Nakamura T, Tokunaga K, Shimomura I, et al. Contribution of visceral fat accumulation to development of coronary artery disease in nonobese men. Atherosclerosis 107: 239-246, 1994.

29. Odamaki M, Furuya R, Kinumura Y, et al. Association between plasma adiponectin concentration and visceral fat accumulation in hemodialysis patients. Nephron Clin Pract 102: c8-c13, 2006.

30. Yamauchi $T$, Kuno $T$, Takada $H$, et al. The impact of visceral fat on multiple risk factors and carotid atherosclerosis in chronic haemodialysis patients. Nephrol Dial Transplant 18: 1842-1847, 2003.

31. Araki T, Emoto M, Yokoyama H, et al. The association of plasma adiponectin level with carotid arterial stiffness. Metabolism 55: 587-592, 2006.

32. Mahmud A, Feely J. Adiponectin and arterial stiffness. Am J Hypertens 18: 1543-1548, 2005.

33. Aso Y, Yamamoto R, Wakabayashi S, et al. Comparison of serum high molecular weight (HMW) adiponectin with total adiponectin concentrations in type 2 diabetic patients with coronary artery disease using an oval enzyme-linked immunosorbent assay to detect HMW adiponectin. Diabetes 55: 1954-1960, 2006.

34. Inoue $\mathrm{T}$, Kotooka $\mathrm{N}$, Morooka $\mathrm{T}$, et al. High molecular adiponectin as a predictor of longterm clinical outcome in patients with coronary artery disease. Am J Cardiol 100: 569-574, 2007.

35. Iwashima $\mathrm{Y}$, Horio $\mathrm{T}$, Kumada $\mathrm{M}$, et al. Adiponectin and renal function, and implications as a risk of cardiovascular disease. Am J Cardiol 98: 1603-1608, 2006.

36. Diez JJ, Iglesias P, Fernandez-Reyes MJ, et al. Serum concentrations of leptin, adiponectin and resistin, and their relationship with cardiovascular disease in patients with end-stage renal disease. Clin Endocrinol 62: 242-249, 2005.

37. Ignacy W, Chudek J, Adamczak M, et al. Reciprocal association of plasma adiponectin and serum C-reactive protein concentration in haemodialysis patients with end-stage kidney disease-a follow-up study. Nephron Clin Pract 101: c18-c24, 2005.

\footnotetext{
(C) 2010 The Japanese Society of Internal Medicine http://www.naika.or.jp/imindex.html
} 